\title{
Non-singular solutions of the Ricci flow on three-manifolds
}

RICHARD S. HAMILTON

\section{Contents.}

1. Classification

2. The lower bound on scalar curvature

3. Limits

4. Long Time Pinching

5. Positive Curvature Limits 701

6. Zero Curvature Limits

7. Negative Curvature Limits 705

8. Rigidity of Hyperbolic metrics

9. Harmonic Parametrizations

10. Hyperbolic Pieces

1. Classification.

In this paper we shall analyze the behavior of all non-singular solutions to the Ricci flow on a compact three-manifold. We consider only essential singularities, those which cannot be removed by rescaling alone. Recall that the normalized Ricci flow ([H1]) is given by a metric $g(x, y)$ evolving 
by its Ricci curvature $R c(x, y)$ with a "cosmological constant" $r=r(t)$ representing the mean scalar curvature:

$$
\frac{\partial}{\partial t} g(X, Y)=2\left[\frac{1}{n} r g(X, Y)-R c(X, Y)\right] .
$$

where

$$
r=\int R / \int 1 .
$$

This differs from the unnormalized flow (without $r$ ) only by rescaling in space and time so that the total volume $V=\int 1$ remains constant.

Definition 1.1. A non-singular solution of the Ricci flow is one where the solution of the normalized flow exists for all time $0 \leq t<\infty$, and the curvature remains bounded $|R m| \leq M<\infty$ for all time with some constant $M$ independent of $t$.

For example, any solution to the Ricci flow on a compact three-manifold with positive Ricci curvature is non-singular, as are the equivariant solutions on torus bundles over the circle found by Isenberg and the author [H-I] which has a homogeneous solution, or the Koiso soliton on a certain four-manifold $[\mathrm{K}]$; by contrast the solutions on a four-manifold with positive isotropic curvature in [H5] definitely become singular, and these singularities must be removed by surgery. Currently there are few conditions which guarantee a solution will remain non-singular; however one may hope to produce nonsingular solutions after a finite number of surgeries.

For any point $P$ let $\rho(P)$ be the injectivity radius at $P$, the largest radius for which the open ball in the tangent space injects under the exponential map; and let $\hat{\rho}$ be the maximum of $\rho(P)$ over all points $P$ in the manifold.

Definition 1.2. We say a solution to the normalized Ricci flow collapses if $\hat{\rho}(t) \rightarrow 0$ as $t \rightarrow \infty$.

In this case it follows from work of Cheeger and Gromov [C-G] that the manifold has an $F$-structure. For three-manifolds this is enough to completely analyze the topology (for example, no counterexample to the Poincaré conjecture can collapse). The torus bundles in [H-I] do collapse.

Now we can state our result.

Theorem 1.3. Any non-singular solution to the normalized Ricci flow on a compact three-manifold does one and only one of the following things: 
C) the solution collapses; or

P) the solution converges to the metric of constant positive sectional curvature; or

Z) the solution converges to a metric of zero sectional curvature; or

$\mathrm{H})$ the solution converges to a metric of constant negative sectional curvature; or

$\left.\mathrm{H}^{\prime}\right)$ we can find a finite collection of complete non-compact hyperbolic three-manifolds with finite volume $\mathcal{H}_{1}, \ldots, \mathcal{H}_{n}$, and for all $t$ beyond some time $T<\infty$ we can find diffeomorphisms $\varphi_{i}(t): \mathcal{H}_{i} \rightarrow \mathcal{M}$ of these manifolds into the manifold $\mathcal{M}$ with the solution so that the pull-back of the solution metric $g(t)$ by $\varphi_{i}(t)$ converges to the hyperbolic metric as $t \rightarrow \infty$; and moreover if we call the exceptional part of $\mathcal{M}$ those points where either the point is not in the image of any $\varphi_{i}$, or where it is but the pull-back metric is not as close to the hyperbolic metric as we like, we can make the volume of the exceptional part as small as we like by taking $t$ large enough; and each $\mathcal{H}_{i}$ is topologically essential in the sense that each $\varphi_{i}$ injects $\pi_{1}\left(\mathcal{H}_{i}\right)$ into $\pi_{1}(\mathcal{M})$.

The important part of case $H^{\prime}$ from the point of view of topology is that $\pi_{1}$ injects; otherwise $\mathcal{M}$ might be something simple like the sphere $S^{3}$ with the hyperbolic metric forming in the complement of a knot while a tube around the knot goes off to infinity. This is enough to answer topological questions (for example, no counterexample to the Poincaré conjecture can contain a hyperbolic piece where $\pi_{1}$ injects). Nevertheless from the perspective of analysis there are still a number of questions we leave unanswered; such as the behavior of the collapse in case $C$, and whether the convergence in cases $Z$ and $H$ (not to mention $H^{\prime}$ ) actually requires modification by a diffeomorphism.

\section{The lower bound on scalar curvature.}

The division of solutions into cases $P, Z$, and $H$ depends on the following remark, which holds in all dimensions.

Theorem 2.1. Let $\check{R}$ be the minimum of the scalar curvature. Under the normalized Ricci flow, whenever $\check{R} \leq 0$ it is increasing; whereas if ever $\check{R} \geq 0$ it remains so forever. 
Proof. The scalar curvature evolves in the normalized flow by the equation

$$
\frac{\partial R}{\partial t}=\Delta R+2\left[|R c|^{2}-\frac{1}{n} r R\right]
$$

as derived in [H1]. If we decompose the Ricci tensor $R c$ into its trace-free part $\stackrel{\circ}{R}$ and its trace $R$, then

$$
|R c|^{2}-\frac{1}{n} r R=|\stackrel{\circ}{R c}|^{2}+\frac{1}{n} R(R-r) .
$$

Now apply the maximum principle. If $\check{R} \leq 0$, then since always $\check{R} \leq r$ (as the minimum must be below the average) we find $\check{R}(\check{R}-r) \geq 0$. Thus if $\check{R} \leq 0$ it must increase. Now if $\check{R} \geq 0$ it cannot go negative again, for when $\check{R}=0$ then $\check{R}(\check{R}-r)=0$ also. Note though that if $\check{R} \geq 0$ then $\check{R}(\check{R}-r)<0$ (unless $R$ is constant), so $\check{R}$ in this case may well decrease.

We can now divide the non-collapsing solutions into Case $P$ where eventually $\check{R}>0$, Case $Z$ where $\lim \check{R}=0$, and Cases $H$ and $H^{\prime}$ where $\lim \check{R}<0$.

\section{Limits.}

Now suppose (again in any dimension) that we have a non-singular solution which does not collapse. Then we can find a sequence of times $t_{j} \rightarrow \infty$ and points $P_{j}$ and some $\delta>0$ so that the injectivity radius of $\mathcal{M}$ at $P_{j}$ in the metric at time $t_{j}$ is at least $\delta$. Then taking the $P_{j}$ as origins and the $t_{j}$ as initial times, by [H6] we can extract a convergent subsequence. However we need some care here, as the limit result in that paper is for solutions to the unnormalized flow. But we can pass back and forth by scaling space and time. So first unnormalize the flow, then take the limit, then normalize again and see what we get. The limit will still satisfy the Ricci flow with a cosmological term $r(t)$, and $r(t)$ for the limit will be the limit of the $r_{j}(t)$ for the sequence. Since we have the curvature bound $\left|R m_{j}\right|_{j} \leq M$ for each term in the sequence ${ }^{1}$ we also have the curvature bound $|R m| \leq M$ in the limit. Since the injectivity radius at $P_{j}$ at time $t_{j}$ is at least $\delta>0$ for all $j$, the injectivity radius of the origin 0 at time 0 in the limit is also at least $\delta>0$. However the volume $\overline{\mathcal{V}}$ of the limit may be different from the constant volume $\mathcal{V}$ of each solution if the diameter goes to infinity; but at least we know $\overline{\mathcal{V}} \leq \mathcal{V}$. The relation of the cosmological term $r(t)$ to the average scalar curvature of the limit is not yet clear.

\footnotetext{
${ }^{1}$ where $R m_{j}(t)=R m\left(t+t_{j}\right)$ and ||$_{j}$ is the norm with respect to the metric $g_{j}(t)=g\left(t+t_{j}\right)$.
} 


\section{Long Time Pinching.}

Here we improve the pinching result in Theorem 24.4 of [H4] (see also Ivey [I].)

Theorem 4.1. Suppose we have a complete solution to the unnormalized Ricci flow on a three-manifold which is complete with bounded curvature for $t \geq 0$. Assume at $t=0$ the eigenvalues $\lambda \geq \mu \geq \nu$ of the curvature operator at each point are bounded below by $\nu \geq-1$. The scalar curvature $R=\lambda+\mu+\nu$ is their sum, and let $X=-\nu$. Then at all points and all times $t \geq 0$ we have the pinching estimate

$$
R \geq X[\ln X+\ln (1+t)-3],
$$

whenever $X>0$.

Proof. This follows as usual by estimating the solutions to the system of ODE's

$$
\left\{\begin{array}{l}
\frac{d \lambda}{d t}=\lambda^{2}+\mu \nu \\
\frac{d \mu}{d t}=\mu^{2}+\lambda \nu \\
\frac{d \nu}{d t}=\nu^{2}+\lambda \mu
\end{array}\right.
$$

from which, if we put $R=\lambda+\mu+\nu$ and $X=-\nu$ and $Y=-\mu$, we get that

$$
\begin{aligned}
& \frac{d X}{d t}=-X^{2}+\lambda Y \\
& \frac{d R}{d t}=\lambda^{2}+Y^{2}+X^{2}+X Y-\lambda X-\lambda Y .
\end{aligned}
$$

Compute

$$
X \frac{d R}{d t}-(X+R) \frac{d X}{d t}=X^{3}+I
$$

where

$$
I=X Y^{2}+\lambda^{2}(X-Y)+\lambda Y(Y-X) .
$$

We claim $I \geq 0$. For if $Y \leq 0$, then $\lambda \geq 0$. Now $I \geq 0$ since $X>0$ and $X-Y \geq 0$. On the other hand, if $Y \geq 0$, then we rewrite $I$ as

$$
I=Y^{3}+(X-Y)\left(\lambda^{2}-\lambda Y+Y^{2}\right) \geq 0 .
$$


Hence

$$
X \frac{d R}{d t}-(X+R) \frac{d X}{d t} \geq X^{3}
$$

Substituting

$$
W=\frac{R}{X}-\ln X
$$

gives

$$
\frac{d W}{d t} \geq X
$$

Now we construct a convex set $\mathcal{Z}$ preserved by the flow of the ODE. $\mathcal{Z}$ is a subset of the product of the space of $3 \times 3$ symmetric matrices (of curvature operators with eigenvalues $\lambda \geq \mu \geq \nu$ ) and the time axis $t$. The set $\mathcal{Z}$ is given by the joint inequalities

$$
R \geq-\frac{3}{1+t}
$$

for all points, and

$$
R \geq X[\ln X+\ln (1+t)-3]
$$

at those points where

$$
X \geq \frac{1}{1+t} .
$$

For any $t$ we get a convex set $\mathcal{Z}(t)$ in the $R$ - $X$ plane. 
The curve and the line meet at an uppermost point. (The set $\mathcal{Z}$ in spacetime need not all be convex, only each time slice.)

It is easy to check that our initial data lies in the given set at $t=0$ since

$$
-1 \leq \nu \leq \mu \leq \lambda
$$

to start, which makes $R \geq-3$ and $X \leq 1$. The lower bound

$$
R \geq-\frac{3}{1+t}
$$

is easily preserved since

$$
\frac{d R}{d t} \geq \frac{2}{3} R^{2} \geq \frac{1}{3} R^{2}
$$

from the ODE. The other bound becomes

$$
W \geq \ln (1+t)-3
$$

which follows easily from

$$
\frac{d W}{d t} \geq X
$$

and the observation that

$$
X \geq \frac{1}{1+t}
$$

on the top boundary curve; since the other side boundary curve is preserved, we could only exit $\mathcal{Z}$ out the top. This proves the theorem.

\section{Positive Curvature Limits.}

Consider the first case where $\check{R}>0$ for large $t$. In this case the maximal time interval $[0, T)$ of the corresponding solution of the unnormalized flow is finite, since

$$
\frac{d}{d t} \check{R} \geq \frac{2}{n} \check{R}^{2}
$$

from the maximum principle. Now for the unnormalized Ricci flow in dimension 3 we have the pinching estimate

$$
R \geq X[\ln X-3]
$$


dropping the time dependence. This assures us that when the curvatures are big, the negative ones are not nearly as large as the positive ones. Now when we rescale to the normalized flow, the scaling factor must go to infinity since $\lim _{t \rightarrow T} \max _{\mathcal{M}}|R m(t)|=\infty$ for the unnormalized flow by Theorem 14.1 of [H1]. In the non-singular case the rescaled positive curvatures stay finite, so the rescaled negative curvatures (if any) go to zero. Thus the limit of the normalized solutions has non-negative sectional curvatures.

Now a complete manifold of non-negative sectional curvature and finite volume must be compact. Then it is proven (see [H2]) that either it is flat, or it splits as a product (or a quotient of a product) of a positively curved surface $S^{2}$ with a circle $S^{1}$, or it has strictly positive curvature. Since a flat three-manifold is a quotient of the three-torus, it cannot have a metric of positive scalar curvature; but our metrics in the sequence do, and since the limit is compact it is the same manifold. The case of a product $S^{2} \times S^{1}$ clearly cannot be non-singular in our sense. Since the limit is compact convergence takes place everywhere, and this makes the cosmological term the same as the average scalar curvature in the limit; thus the limit is also a non-singular solution of the normalized flow in this case, which rules out $S^{2} \times S^{1}$. The remaining case of strictly positive sectional curvature is the only one that can occur in the limit. Thus the limit of the solutions around $\left(P_{j}, t_{j}\right)$ is one which itself goes to constant positive curvature as the new time $t \rightarrow \infty$. But then the original solution did also by [H1]. This finishes the case $P$ of positive curvature.

\section{Zero Curvature Limits.}

Next consider the case $\check{R} \rightarrow 0$ as $t \rightarrow \infty$; this is the case $Z$ of zero curvature. Again we can take a limit around points $P_{j}$ as origins and times $t_{j}$ as initial times whenever the injectivity radius at all $P_{j}$ at times $t_{j}$ is at least some fixed $\delta>0$. Since $\check{R} \rightarrow 0$ for each solution in the sequence, the limit has $\check{R} \geq 0$; likewise since $r \geq \check{R}$ for each solution in the sequence, the limit has $r \geq 0$ also. We want to claim this limit has non-negative sectional curvature. If this is true we will be done in the same way as the positive curvature case. When we want to use our pinching estimates, we can rescale the initial data to have all sectional curvatures bounded in absolute value by one.

To see the limit has non-negative sectional curvature, consider the unnormalized flow of the original solution. Its volume $\mathcal{V}$ now changes. We consider three cases. First suppose there is a sequence of times $t_{j} \rightarrow \infty$ 
where $\mathcal{V}\left(t_{j}\right) \rightarrow \infty$. In this case, because

$$
\frac{d \mathcal{V}}{d t}=-r \mathcal{V}
$$

we find

$$
\ln \frac{\mathcal{V}\left(t_{j}\right)}{\mathcal{V}(0)}=-\int_{0}^{t_{j}} r(t) d t
$$

and we would get a contradiction unless there were another sequence of times $\tilde{t}_{k} \rightarrow \infty$ when $r\left(\tilde{t}_{k}\right) \leq 0$. Choose points $\tilde{P}_{k}$ at these times $\tilde{t}_{k}$ to take our limit of the normalized flow. Normalizing by a factor does not change $r\left(\tilde{t}_{k}\right) \leq 0$. On the other hand $r\left(\tilde{t}_{k}\right) \geq \check{R}\left(\tilde{t}_{k}\right)$ and $\check{R} \rightarrow 0$ as $t \rightarrow \infty$ for the normalized flow; so to summarize

$$
r\left(\tilde{t}_{k}\right) \rightarrow 0 \quad \text { and } \quad \check{R}\left(\tilde{t}_{k}\right) \rightarrow 0 \quad \text { as } \quad k \rightarrow \infty
$$

for the normalized flow. Now

$$
\int R-\check{R}=\int r-\check{R}=[r-\check{R}] \mathcal{V}
$$

and $\mathcal{V}$ is constant for the normalized flow. This gives

$$
\int R-\check{R} \rightarrow 0 \text { as } k \rightarrow \infty
$$

at the sequence of times $\tilde{t}_{k} \rightarrow \infty$. Note that $R-\check{R} \geq 0$. As we take the limit of the sequence we may be left with only part of the manifold (when the limit is noncompact,) but this does not matter; we still get

$$
\int R=0
$$

for the limit of the normalized solutions at $t=0$. But $R \geq 0$, so $R=0$. Now consider the unnormalized limit flow; we still have $R \geq 0$ for all $t$ and $R=0$ at $t=0$. By the strong maximum principle applied to

$$
\frac{\partial R}{\partial t}=\Delta R+2|R c|^{2}
$$

this can only happen if the metric is flat, since the limit flow exists for $-\infty<t<\infty$. 
The second case is where for the unnormalized flow we can find a sequence of times $t_{j} \rightarrow \infty$ when $\mathcal{V}\left(t_{j}\right) \rightarrow 0$. In this case before we rescale all the eigenvalues of the curvature operator lie in the region where

$$
R \geq X[\ln X-3]
$$

(neglecting the improvement with time). Pick a sequence of points $P_{j}$ where the injectivity radius is at least some $\delta>0$ at time $t_{j}$ for all $j \rightarrow \infty$. Since $\mathcal{V}\left(t_{j}\right) \rightarrow 0$, when we rescale we expand, which reduces curvature. We claim the limit has non-negative sectional curvature. For if the maximum value of $X$ does not go to infinity in the unnormalized flow, it must get rescaled to zero to control the volume; while if the maximum value of $X$ does go to infinity, in the unnormalized flow the maximum value of $R$ will go to infinity even faster from the pinching estimate, and when we rescale we keep $R$ bounded so $X$ will go to zero in this case also. Thus in either case the limit has non-negative sectional curvature.

The third and final case is where the volume $\mathcal{V}$ for the unnormalized flow is bounded above and below as $t \rightarrow \infty$ by

$$
0<c \leq \mathcal{V} \leq C<\infty
$$

for some constants $c$ and $C$. In this case normalizing the flow only changes quantities in a bounded way. Now we can use the time-improved pinching estimate

$$
R \geq X[\ln X+\ln (1+t)-3] .
$$

There are two cases. If

$$
X \leq \frac{A}{1+t}
$$

for any constant $A$ then $X \rightarrow 0$ as $t \rightarrow \infty$ and the limit has non-negative curvature. On the other hand if we can pick a sequence of times $t_{j} \rightarrow \infty$ and points $P_{j}$ where $X_{j}=\max X$ at time $t_{j}$ satisfies

$$
X_{j}\left(1+t_{j}\right) \rightarrow \infty
$$

then when $R_{j}=R\left(P_{j}\right)$ we have

$$
R_{j} / X_{j} \rightarrow \infty
$$

from the time-improved pinching estimate

$$
\frac{R}{X} \geq \ln [X(1+t)]-3 .
$$


But $R_{j} \leq C$ since we don't need to normalize, and this shows $X_{j} \rightarrow 0$. Thus the limit has non-negative sectional curvature in this case as in all the others.

\section{Negative Curvature Limits.}

Finally we come to the interesting case where $\check{R}$ increases monotonically to a limit strictly less than zero. By scaling we can assume $\check{R} \rightarrow-3$. Now take any sequence of points $P_{j}$ and times $t_{j}$ where the injectivity radii are all at least some $\delta>0$, and take the limit; we call this a non-collapsing limit.

Lemma 7.1. In the negative case $\check{R} \rightarrow-3$ all non-collapsing limits are hyperbolic with constant curvature $\lambda=\mu=\nu=-1$.

Proof. Recall the evolution equation for $R$

$$
\frac{\partial}{\partial t} R=\Delta R+2|\stackrel{\circ}{R c}|^{2}+\frac{2}{3} R(R-r) .
$$

from which we get the ordinary differential inequality on $\check{R}$ that

$$
\frac{d}{d t} \check{R} \geq \frac{2}{3} \check{R}(\check{R}-r) \text {. }
$$

Since $\check{R} \leq-3$ we have

$$
\frac{d}{d t} \check{R} \geq 2(r-\check{R})
$$

This makes

$$
\int_{0}^{\infty}(r-\check{R}) d t<\infty
$$

which forces $r$ to be near -3 most of the time. In fact for any $\varepsilon>0$ we can find a time $T$ so that for $t \geq T$

$$
0 \leq \int_{t}^{t+1}(r-\check{R}) d t \leq \varepsilon .
$$

We can also make $\check{R}$ lie in the interval

$$
-3-\varepsilon \leq \check{R} \leq-3
$$


for $t \geq T$, and then

$$
-\varepsilon \leq \int_{t}^{t+1}(r+3) d t \leq \varepsilon .
$$

When we pass to the limit, the cosmological factor $r(t)$ on the limit satisfies

$$
\int_{t}^{t+1}(r+3) d t=0
$$

on every time interval, and thus $r \equiv-3$ on the limit.

Now going back to the flow before we take a limit, we have

$$
\int R=\int r
$$

since $r$ is the average of $R$. Also

$$
|R-r|=|(R-\check{R})-(r-\check{R})| \leq(R-\check{R})+(r-\check{R}) .
$$

Integrating over the manifold

$$
\int|R-r| d v \leq \int(R-\check{R}) d v+\int(r-\check{R}) d v
$$

which gives

$$
\int|R-r| d v \leq 2(r-\check{R}) \mathcal{V}
$$

Integrating in time as well gives

$$
\iint|R-r| d v d t<\infty
$$

When we pass to the limit of a sequence $t_{j} \rightarrow \infty$ of translates we find

$$
\iint|R-r| d v d t=0
$$

for the limit flow, which makes $R \equiv r \equiv-3$. Now the strong maximum principle applied to the evolution of $R$ shows $|\stackrel{\circ}{R c}|=0$ as well. Thus the limit metric has constant curvature $\lambda=\mu=\nu=-1$ as claimed. 


\section{Rigidity of Hyperbolic metrics.}

We can define the topology of $C^{\infty}$ convergence on compact sets for tensors on a Riemannian manifold by the seminorms $C^{k}(\mathcal{K})$ for compact sets $\mathcal{K}$ and integers $k$, where for a tensor $T$ with covariant derivatives $D^{i} T$ we have

$$
\|T\|_{C^{k}(\mathcal{K})}=\sum_{i=1}^{k} \sup _{\mathcal{K}}\left|D^{i} T\right| .
$$

We can also define the topology of $C^{\infty}$ convergence on compact sets for maps $F: \mathcal{M} \rightarrow \mathcal{N}$ of one Riemannian manifold to another. For any compact set $\mathcal{K}$ in $\mathcal{M}$ and any two maps $F$ and $G$ put

$$
d_{\mathcal{K}}(F, G)=\sup _{X \in \mathcal{K}} d(F(X), G(X))
$$

where $d(Y, Z)$ is the geodesic distance from $Y$ to $Z$ on $\mathcal{N}$. Recall that the space $J^{k} \mathcal{M}$ of $k$-jets of paths on $\mathcal{M}$ is the collection of all

$$
\left(P, J^{1}, J^{2}, \ldots, J^{k}\right)
$$

where $P$ is a point on $\mathcal{M}$ and $J^{i}$ is a tangent vector for $1 \leq i \leq k$; if $P(t)$ is a path in $\mathcal{M}$ parametrized by $t$ then the $i$-th covariant derivative

$$
\frac{d^{i} P}{d t^{i}}=J^{i}
$$

is its $i$-jet, so that $J^{1}$ is the velocity and $J^{2}$ is the acceleration, and so on. A map $F: \mathcal{M} \rightarrow \mathcal{N}$ clearly induces a map $J^{k} F: J^{k} \mathcal{M} \rightarrow J^{k} \mathcal{N}$ called its $k$-jet extension. Consider the unit ball $\mathcal{B} J^{k} \mathcal{K}$ of all $k$-jets $\left(P, J^{1}, \ldots, J^{k}\right)$ with $P \in \mathcal{K}$ and

$$
\left|J^{1}\right|^{2}+\left|J^{2}\right|^{2}+\cdots+\left|J^{k}\right|^{2} \leq 1 .
$$

Define the $k$-jet distance between $F$ and $G$ on the set $\mathcal{K}$ to be

$$
d_{C^{k}(\mathcal{K})}(F, G)=d_{\mathcal{B J}^{k} \mathcal{K}}\left(J^{k} F, J^{k} G\right) .
$$

Then convergence in the metrics $C^{k}(\mathcal{K})$ for all $k$ and $\mathcal{K}$ defines the topology of $C^{\infty}$ convergence on compact sets for the space of maps.

Now we can state the basic rigidity results we will use. These results go back to work of Mostow. 
8.1 Rigidity of Hyperbolic Manifolds. If a map of a large enough part of one complete hyperbolic manifold with finite volume into another with no fewer cusps is close enough to being an isometry, then there exists an actual isometry between the manifolds. Specifically, for any complete hyperbolic manifold $\mathcal{H}$ with finite volume with metric $h$, we can find a compact subset $\mathcal{K}$ of $\mathcal{H}$, an integer $k$ and $a \delta>0$ with the following property; if $F$ is a map of $\mathcal{K}$ into another complete hyperbolic manifold $\widetilde{\mathcal{H}}$ with no fewer cusps, finite volume and metric $\tilde{h}$ such that

$$
\left\|F^{*} \widetilde{h}-h\right\|_{C^{k}(\mathcal{K})}<\delta
$$

then there is an isometry $I$ of $\mathcal{H}$ to $\widetilde{\mathcal{H}}$.

8.2 Rigidity of Isometries. For any complete hyperbolic manifold with finite volume $\mathcal{H}$ and metric $h$, we can find a compact subset $\mathcal{K}$ of $\mathcal{H}$ with the following property; if $F$ is an isometry of $\mathcal{K}$ into $\mathcal{H}$, so that $F^{*} h=h$ on $\mathcal{K}$, then there exists a global isometry $I$ of $\mathcal{H}$ to itself with $F=I$ on $\mathcal{K}$.

We can combine these results in the following corollary.

8.3 General Rigidity. For any complete hyperbolic manifold $\mathcal{H}$ with finite volume with metric $h$ we can find a compact set $\mathcal{K}$ so that, for every integer $\ell$ and every $\varepsilon>0$ we can find an integer $k$ and $a \delta>0$ with the following property; if $F$ is a map of $\mathcal{K}$ into another complete hyperbolic manifold $\tilde{\mathcal{H}}$ with no fewer cusps (than $\mathcal{H}$,) finite volume with metric $\tilde{h}$ such that

$$
\left\|F^{*} \widetilde{h}-h\right\|_{C^{k}(\mathcal{K})}<\delta
$$

then there exists an isometry $I$ of $\mathcal{H}$ to $\tilde{\mathcal{H}}$ such that

$$
d_{C^{\ell}(\mathcal{K})}(F, I)<\varepsilon .
$$

Proof. First we can require that $\mathcal{K}, k$ and $\delta$ be chosen well enough from the theorem of Rigidity of Hyperbolic Manifolds to guarantee that $\mathcal{H}$ and $\widetilde{\mathcal{H}}$ are isometric by an isometry $I$, and with $\mathcal{K}$ large enough so that we also have the result on rigidity of isometries. Then we can assume $\widetilde{\mathcal{H}}=\mathcal{H}$. For this $\mathcal{K}$, suppose there is some $\ell$ and $\varepsilon>0$ so that no matter how large we take $k$ or how small we take $\delta>0$ we can still find $F$ mapping $\mathcal{K}$ into $\mathcal{H}$ with

$$
\left\|F^{*} h-h\right\|_{C^{k}(\mathcal{K})}<\delta
$$


and

$$
d_{C^{\ell}(\mathcal{K})}(F, I) \geq \varepsilon
$$

for all isometries $I$ of $\mathcal{H}$ to itself. Taking a sequence $k_{j} \rightarrow \infty$ and $\delta_{j} \rightarrow 0$, we can find such a sequence of maps $F_{j}$ and extract a subsequence convergent to a map $F_{\infty}$ with $F_{\infty}^{*} h=h$ on $\mathcal{K}$. Then there exists a global isometry $I$ of $\mathcal{H}$ to itself with $F_{\infty}=I$ on $\mathcal{K}$. For this $I$

$$
d_{C^{\ell}(\mathcal{K})}\left(F_{j}, I\right)<\varepsilon
$$

when $j$ is large enough contradicting our assumption. This proves the corollary.

\section{Harmonic Parametrizations.}

In order to show that hyperbolic pieces persist in the solution as $t \rightarrow \infty$, we will need to use a special parametrization given by harmonic maps.

Theorem 9.1. Let $\mathcal{M}$ be a compact Riemannian manifold with boundary with strictly negative Ricci curvature and strictly concave boundary. Then for every metric $\widetilde{g}$ on $\mathcal{M}$ close enough to the original metric $g$ we can find a unique (among maps close to the identity) diffeomorphism $F$ of $\mathcal{M}$ to itself so that $F:(\mathcal{M}, g) \rightarrow(\mathcal{M}, \widetilde{g})$ is harmonic and $F$ takes the boundary $\partial \mathcal{M}$ to itself and satisfies the free boundary condition that the normal derivative $D_{N} F$ of $F$ at the boundary is normal to the boundary.

Proof. We apply the inverse function theorem. Let $\Phi(\mathcal{M}, \partial \mathcal{M})$ be the space of maps of $\mathcal{M}$ to itself which take $\partial \mathcal{M}$ to itself. Then $\Phi(\mathcal{M}, \partial \mathcal{M})$ is a manifold (indeed a Banach manifold in an appropriate norm such as $C^{2+\alpha}$ or $L_{2}^{1}$ ) and the tangent space to $\Phi(\mathcal{M}, \partial \mathcal{M})$ at the identity is the space of vector fields $V=V^{i} \frac{\partial}{\partial x^{i}}$ tangent to the boundary. Consider the map sending $F \in \Phi(\mathcal{M}, \partial \mathcal{M})$ to the pair $\left\{\Delta F,\left(D_{N} F\right)_{\|}\right\}$consisting of the harmonic map Laplacian and the tangential component (in the target) of the normal derivative of $F$ at the boundary. We only need to check that the derivative of this map is an isomorphism at the identity. The resulting operator is an elliptic boundary value problem whose kernel is the space of solutions of

$$
\begin{aligned}
\Delta V+R c(V)=0 & & \text { on } \mathcal{M} \\
V_{\perp}=0 & & \text { at } \partial \mathcal{M} \\
\left(D_{N} V\right)_{\|}-\Pi(V)=0 & & \text { at } \partial \mathcal{M}
\end{aligned}
$$


where $V_{\perp}$ is the normal component of $V,\left(D_{N} V\right)_{\|}$is the tangential component of the normal derivative of $V, R c$ is the Ricci tensor (as an automorphism of $T \mathcal{M}$ ) and $\Pi$ is the second fundamental form of the boundary (as an automorphism of $T \partial \mathcal{M}$ ). Notice that Killing vector fields tangent to the boundary automatically satisfy these equations.

Now using these equations and integrating by parts gives

$$
\iint_{\mathcal{M}}|D V|^{2}=\int_{\partial \mathcal{M}} R c(V, V)+\int_{\partial \mathcal{M}} \Pi(V, V) .
$$

If $R c<0$ and $\Pi<0$ we conclude that the kernel is trivial. Since this elliptic boundary value problem is self-adjoint (because of the free boundary condition) the cokernel is trivial also. This proves the theorem.

Definition 9.2. Let $\mathcal{K}$ be a compact manifold with boundary $\partial \mathcal{K}$ and metric $h$, and let $\mathcal{M}$ be a compact manifold without boundary and metric $g$. We say a diffeomorphism $F$ from $\mathcal{K}$ into $\mathcal{M}$ satisfies the constant mean curvature boundary conditions if

(1) $F(\partial \mathcal{K})$ is a constant mean curvature hypersurface in $\mathcal{M}$, and

(2) the area of each component of $F(\partial \mathcal{K})$ equals the area of the corresponding component of $\partial \mathcal{K}$, and

(3) the normal derivative of $F$ at $\partial \mathcal{K}$ is normal to the boundary image $F(\partial \mathcal{K})$.

Theorem 9.3. Let $\mathcal{K}$ be a compact manifold with a metric $h$ of strictly negative Ricci curvature and a strictly concave boundary. Then there exists a number $k$ and $a$ constant $\delta>0$ depending only on $\mathcal{K}$ and $h$ with the following property. If $\mathcal{M}$ is a compact manifold with a one-parameter family of metrics $g_{t}$ for $\alpha \leq t \leq \beta$, and if at $t=\alpha$ we can find a harmonic diffeomorphism $F_{\alpha}$ of $\mathcal{K}$ into $\mathcal{M}$ with metric $g_{\alpha}$ satisfying the constant mean curvature boundary conditions and with

$$
\left\|F_{\alpha}^{*} g_{\alpha}-h\right\|_{C^{k}(\mathcal{K})} \leq \delta
$$

then we can extend $F_{\alpha}$ to a one-parameter family $F_{t}$ of harmonic diffeomorphisms of $\mathcal{K}$ into $\mathcal{M}$ with metric $g_{t}$ satisfying the constant mean curvature boundary conditions and varying smoothly in $t$ with

$$
\left\|F_{t}^{*} g_{t}-h\right\|_{C^{k(\mathcal{K})}} \leq \delta
$$


on a maximal interval $\alpha \leq t \leq \omega$; and either $\omega=\beta$ or else

$$
\left\|F_{\omega}^{*} g_{\omega}-h\right\|_{C^{k(\mathcal{K})}}=\delta \text {. }
$$

Proof. Suppose at some time $t=\pi$ we have a harmonic diffeomorphism $F_{\pi}$ of $\mathcal{K}$ into $\mathcal{M}$ with metric $g_{\pi}$ satisfying the constant mean curvature boundary conditions. By the inverse function theorem we can first find a constant mean curvature hypersurface near $F_{\pi}(\partial \mathcal{K})$ in $\mathcal{M}$ with the metric $g_{t}$ for $t$ close to $\pi$ and with the same area for each component; note that since $\partial \mathcal{K}$ is strictly concave and $F_{\pi}$ is close to being an isometry we know $F_{\pi}(\partial \mathcal{K})$ is also strictly concave, and the foliation of a neighborhood of $F_{\pi}(\partial \mathcal{K})$ by constant mean curvature hypersurfaces has the area as a function with nonzero gradient, which allows us to adjust the area as we choose. Then by the inverse function theorem again in Theorem 9.1, we can find the harmonic diffeomorphism $F_{t}$ near $F_{\pi}$ which takes $\partial \mathcal{K}$ into the constant mean curvature hypersurface we just found and also satisfies the free boundary condition. Thus the set of $t$ where we can extend the map as desired (except for the norm inequality) is open. Next we show it is closed also.

Suppose then we have a diffeomorphism $F_{t}$ such as we desire for $\alpha \leq t<\omega$. We claim we can take the limit of $F_{t}$ as $t \rightarrow \omega$ to get the map we want at $\omega$. Note that the $F_{t}$ satisfy

$$
\left\|F_{t}^{*} g_{t}-h\right\|_{C^{k}(\mathcal{K})} \leq \delta
$$

and the metrics $g_{t}$ for $\alpha \leq t \leq \beta$ are uniformly equivalent; this implies that the $F_{t}$ are equicontinuous, and we can find a subsequence $t_{j} \rightarrow \omega$ for which the $F_{t}$ converge uniformly to a map $F_{\omega}$. We can then in fact choose a further subsequence so that the $F_{t}$ converge to $F_{\omega}$ in $C^{k-1}(\mathcal{K})$, because the bounds in $C^{k}$ imply equicontinuity in $C^{k-1}$. The the limit map has

$$
\left\|F_{\omega}^{*} g_{\omega}-h\right\|_{C^{k-1}(\mathcal{K})} \leq \delta .
$$

We need to check that $F_{\omega}$ is still a diffeomorphism. We at least know $F_{\omega}$ is a local diffeomorphism, and $F_{\omega}$ is the limit of diffeomorphisms $F_{t_{j}}$, so the only possibility of overlap is at the boundary. Here we use the fact that we know $F_{\omega}(\partial \mathcal{K})$ is still strictly concave if $k$ is large and $\delta$ is small, and this prevents the boundary from touching itself. Thus $F_{\omega}$ is a diffeomorphism.

A limit of harmonic maps is harmonic, so $F_{\omega}$ is a harmonic diffeomorphism from $\mathcal{K}$ into $\mathcal{M}$ with the metric $g_{\omega}$. A limit of constant mean curvature hypersurfaces with the same area is again a constant mean curvature hypersurface with the same area, so $F_{\omega}(\partial \mathcal{K})$ has constant mean curvature and 
the same area as $\partial \mathcal{K}$. Likewise $F_{\omega}$ continues to satisfy the free boundary condition that the normal derivative of $F_{\omega}$ at $\partial \mathcal{K}$ is normal to $F_{\omega}(\partial \mathcal{K})$.

We can now use our previous argument, where $k-1$ suffices in place of $k$ if we take $k$ one bigger to start, to see that $F_{\omega}$ extends to harmonic diffeomorphisms $F_{t}$ for $t$ near $\omega$ satisfying the constant mean curvature boundary conditions. Then the uniqueness part of the inverse function theorem in Theorem 9.1 guarantees that the previous family $F_{t}$ for $\alpha \leq t<\omega$ agrees with this new family around $\omega$ where they overlap. This makes $F_{\omega}$ the limit of the $F_{t}$ in $C^{\infty}(\mathcal{K})$, not just in $C^{k-1}(\mathcal{K})$. Now we get

$$
\left\|F_{\omega}^{*} g_{\omega}-h\right\|_{C^{k}(\mathcal{K})} \leq \delta
$$

as desired. This allows us to continue the family $F_{t}$ until $\omega=\beta$ or

$$
\left\|F_{\omega}^{*} g_{\omega}-h\right\|_{C^{k}(\mathcal{K})}=\delta
$$

which finishes the proof.

Recall that a sequence of manifolds $\mathcal{M}_{j}$ with metrics $g_{j}$ and origins $P_{j}$ and orthonormal frames $\mathcal{F}_{j}$ at $P_{j}$ converges to a complete manifold $\mathcal{M}$ with metric $g$ and origin $P$ and frame $\mathcal{F}$ if we can find a sequence of compact sets $\mathcal{K}_{j}$ in $\mathcal{M}$ exhausting $\mathcal{M}$ and a sequence of diffeomorphisms $F_{j}$ of neighborhoods of $\mathcal{K}_{j}$ into $\mathcal{M}_{j}$ so that $F_{j}$ takes the origin $P$ to the origin $P_{j}$ and the tangent map $T F$ takes the frame $\mathcal{F}$ to the frame $\mathcal{F}_{j}$, and so that

$$
\left\|F_{j}^{*} g_{j}-g\right\|_{C^{k}(\mathcal{K})} \rightarrow 0
$$

as $j \rightarrow \infty$ for all compact $\mathcal{K}$ in $\mathcal{M}$ and all integers $k$.

We can extend the metric $g$ on $\mathcal{M}$ to a metric on the orthonormal frame bundle using the connection to define horizontal and vertical subspaces, and using the usual metric on the orthogonal group which appears as the fibres. This allows us to define the distance between frames. Now we state a result which lets us replace the approximating maps in the limit of a sequence of manifolds with ones chosen systematically, at least in our case.

Theorem 9.4. Let $\mathcal{M}_{j}$ be a sequence of manifolds with metrics $g_{j}$ and origins $P_{j}$ and frames $\mathcal{F}_{j}$ which converge to a complete manifold $\mathcal{M}$ with metric $g$ and origin $P$ and frame $\mathcal{F}$. Suppose the limit $\mathcal{M}$ contains a compact subset $\mathcal{K}$ where the Ricci curvature is strictly negative, and $\mathcal{K}$ has a smooth boundary $\partial \mathcal{K}$ which is strictly concave, and $\mathcal{K}$ contains the origin $P$. Then we can find a sequence of maps $F_{j}$ from $\mathcal{K}$ into $\mathcal{M}_{j}$ for $j$ which are harmonic diffeomorphisms and satisfy the constant mean curvature boundary conditions, 
and which have $d\left(F_{j}(P), P_{j}\right) \rightarrow 0$ and $d\left(T F_{j}(\mathcal{F}), \mathcal{F}_{j}\right) \rightarrow 0$ as $j \rightarrow \infty$, and also have

$$
\left\|F_{j}^{*} g_{j}-g\right\|_{C^{k}(\mathcal{K})} \rightarrow 0
$$

as $j \rightarrow \infty$ for all integers $k$.

Proof. Since $\partial \mathcal{K}$ is strictly concave, we can foliate a neighborhood of $\partial \mathcal{K}$ with constant mean curvature hypersurfaces where the area $A$ has a nonzero gradient. When the approximating maps $F_{j}: \mathcal{K} \subseteq \mathcal{M} \rightarrow \mathcal{M}_{j}$ are close enough to isometries on this collar of $\partial \mathcal{K}$, the metrics $g_{j}$ on $\mathcal{M}_{j}$ will also admit nearby constant mean curvature hypersurfaces foliating a neighborhood of $\partial \mathcal{K}$, by the inverse function theorem. Since the area $A$ on $\mathcal{M}_{j}$ still has non-zero gradient, one of these will have the same area as $\partial \mathcal{K}$. Moreover we can change the map $F_{j}$ by an amount which goes to zero as $j \rightarrow \infty$ so that now $F_{j}(\partial \mathcal{K})$ is this constant mean curvature hypersurface. The pull-back metric $F_{j}^{*} g_{j}$ will be as close as we like to $g$, so we can again change $F_{j}$ by an amount which goes to zero as $j \rightarrow \infty$ so as to make $F_{j}$ a harmonic diffeomorphism and satisfy the constant mean curvature boundary conditions, by the inverse function theorem result in Theorem 9.1. Before we modified $F_{j}$ we had $F_{j}(P)=P_{j}$ and $T F_{j}(\mathcal{F})=\mathcal{F}_{j}$, and we only change $F_{j}$ by an amount going to zero; so after the change we have

$$
d\left(F_{j}(P), P_{j}\right) \rightarrow 0 \quad \text { and } \quad d\left(T F_{j}(\mathcal{F}), \mathcal{F}_{j}\right) \rightarrow 0
$$

as desired. Also since we change $F_{j}$ by as little as we wish on $\mathcal{K}$, we still have

$$
\left\|F_{j}^{*} g_{j}-g\right\|_{C^{k}(\mathcal{K})} \rightarrow 0
$$

for all $k$.

\section{Hyperbolic Pieces.}

Suppose $\mathcal{H}$ is a complete hyperbolic manifold with finite volume. For all small enough $A>0$ we can truncate each cusp along a constant mean curvature torus of area $A$ which is uniquely determined; the remainder we denote by $\mathcal{H}_{A}$. As $A \rightarrow 0$ the $\mathcal{H}_{A}$ exhaust $\mathcal{H}$. The choice of $A$ does not depend on $\mathcal{H}$. If $\varepsilon_{0}>0$ is the Margulis constant, then once we are in a cusp at a point with injectivity radius less than $\varepsilon_{0}$, we stay in that cusp as 
long the injectivity radius is less than $\varepsilon_{0}$. Hence we can truncate cusps at constant curvature tori of area $A \leq A_{0}=c \varepsilon_{0}^{2}$ where $c$ is a universal constant.

Now we prove the persistence of hyperbolic pieces. Consider all the possible hyperbolic limits of a given solution to the Ricci flow, and among them choose one $\mathcal{H}$ with the least possible number of cusps. Suppose the times $t_{j}$ have the manifold $\mathcal{M}$ with the metrics $g\left(t_{j}\right)$ and origins $P_{j}$ and frames $\mathcal{F}_{j}$ converge to $\mathcal{H}$ with hyperbolic metric $h$ and origin $P$ and frame $\mathcal{F}$. Pick a number $A>0$ to truncate cusps, an integer $\ell$ and an $\varepsilon$ sufficient to guarantee from Theorem 9.1 the uniqueness of the identity map $I$ among maps close to $I$ as a harmonic map $F$ from $\mathcal{H}_{A}$ with the given hyperbolic metric $h$ to itself where $F$ satisfies the boundary conditions of taking $\partial \mathcal{H}_{A}$ to $\partial \mathcal{H}_{A}$ and with the normal derivative of $F$ at the boundary of the domain normal to the boundary of the target. Then choose $k$ and $\delta>0$ from Theorem 8.3 on rigidity.

By Theorem 9.4 we can guarantee for all large enough $j$ the existence of harmonic diffeomorphisms $F_{j}$ of $\mathcal{H}_{A}$ to a manifold $\mathcal{M}_{A}\left(t_{j}\right)$ obtained by truncating $\mathcal{M}$ with metric $g\left(t_{j}\right)$ at constant mean curvature tori of area $A$, with $F_{j}$ taking $\partial \mathcal{H}_{A}$ to $\partial \mathcal{M}_{A}\left(t_{j}\right)$ and normal to the boundary at the boundary as above. Since the metric $g(t)$ varies smoothly with $t_{j}$, by Theorem 9.3 we can smoothly continue the map $F_{j}$ to a family $F_{j}(t)$ with $F_{j}\left(t_{j}\right)=F_{j}$ and with $F_{j}(t)$ having all the above properties. If for some $j$ we can continue all the way as $t \rightarrow \infty$, the hyperbolic piece persists. Otherwise we get a contradiction as follows.

For each $j$ large enough, we can continue the family $F_{j}(t)$ for $t_{j} \leq t \leq t_{j}^{\#}$ where $t_{j}^{\#}$ is the first time when

$$
\left\|F_{j}(t)^{*} g(t)-h\right\|_{C^{k}\left(\mathcal{H}_{A}\right)}=\delta
$$

by Theorem 9.3. Consider the new sequence of the manifolds $\mathcal{M}$ with metrics $g\left(t_{j}^{\#}\right)$ and origins $F_{j}\left(t_{j}^{\#}\right) P$ and frames $T F_{j}\left(t_{j}^{\#}\right) \mathcal{F}$. Since the $F_{j}\left(t_{j}^{\#}\right)$ are close to isometries, the injectivity radii of the metrics $g\left(t_{j}^{\#}\right)$ at $F_{j}\left(t_{j}^{\#}\right) P$ do not go to zero, and we can extract a subsequence which again converges to a hyperbolic limit $\widetilde{\mathcal{H}}$ with metric $\tilde{h}$ and origin $\widetilde{P}$ and frame $\widetilde{\mathcal{F}}$. The new limit $\widetilde{\mathcal{H}}$ has at least as many cusps as the old limit $\mathcal{H}$, since we chose $\mathcal{H}$ with as few as possible.

To simplify the notation, write $g_{j}^{\#}=g\left(t_{j}^{\#}\right), F_{j}^{\#}=F_{j}\left(t_{j}^{\#}\right), P_{j}^{\#}=F_{j}\left(t_{j}^{\#}\right) P$ and $\mathcal{F}_{j}^{\#}=T F_{j}\left(t_{j}^{\#}\right) \mathcal{F}$. Then the maps $F_{j}^{\#}$ are harmonic diffeomorphisms of $\mathcal{H}_{A}$ with metric $h$ into $\mathcal{M}$ with metrics $g_{j}^{\#}$ satisfying the constant mean curvature boundary conditions with $F_{j}^{\#} P=P_{j}^{\#}$ and $T F_{j}^{\#} \mathcal{F}=\mathcal{F}_{j}^{\#}$, while 
the sequence of manifolds $\mathcal{M}$ with metrics $g_{j}^{\#}$ and origins $P_{j}^{\#}$ and frames $\mathcal{F}_{j}^{\#}$ converges as $j \rightarrow \infty$ to the manifold $\tilde{\mathcal{H}}$ with metric $\tilde{h}$ and origin $\widetilde{P}$ and frame $\tilde{\mathcal{F}}$. We also know that

$$
\left\|\left(F_{j}^{\#}\right)^{*} g_{j}^{\#}-h\right\|_{C^{k}(\mathcal{K})}=\delta
$$

for each $j$.

By the definition of convergence, we can find a sequence of compact sets $\widetilde{\mathcal{K}}_{j}$ exhausting $\widetilde{\mathcal{H}}$ and containing $\widetilde{P}$, and diffeomorphisms $\widetilde{F}_{j}$ of neighborhoods of $\widetilde{\mathcal{K}}_{j}$ into $\mathcal{M}$ with $\widetilde{F}_{j} \widetilde{P}=P_{j}^{\#}$, and $T \widetilde{F}_{j} \widetilde{\mathcal{F}}=\mathcal{F}_{j}^{\#}$ such that for each compact set $\widetilde{\mathcal{K}}$ in $\widetilde{\mathcal{H}}$ and each integer $\widetilde{k}$

$$
\left\|\widetilde{F}_{j}^{*} g_{j}^{\#}-\widetilde{h}\right\|_{C^{\widetilde{k}}(\widetilde{\mathcal{K}})} \rightarrow 0
$$

as $j \rightarrow \infty$. For large enough $j$ the sets $\widetilde{F}_{j}\left(\widetilde{\mathcal{K}}_{j}\right)$ will contain all the points out to any fixed distance we need from $P_{j}^{\#}$; and hence

$$
\widetilde{F}_{j}\left(\widetilde{\mathcal{K}}_{j}\right) \supseteq F_{j}^{\#}\left(\mathcal{H}_{A}\right)
$$

since $\mathcal{H}_{A}$ is at bounded distance from $P$ and $F_{j}^{\#} P_{j}=P_{j}^{\#}$ and $F_{j}^{\#}$ is reasonably close to preserving the metrics. Then we can form the composition

$$
H_{j}=\widetilde{F}_{j}^{-1} \circ F_{j}^{\#}: \mathcal{H}_{A} \rightarrow \tilde{\mathcal{H}} .
$$

Since the $\widetilde{F}_{j}$ are as close to preserving the metric as we like, for any $\tilde{\delta}>\delta$ we have

$$
\left\|H_{j}^{*} \widetilde{h}-h\right\|_{C^{k}\left(\mathcal{H}_{A}\right)}<\widetilde{\delta}
$$

for large enough $j$. Then a subsequence of $H_{j}$ converge at least in $C^{k-1}\left(\mathcal{H}_{A}\right)$ to a map $H_{\infty}$ of $\mathcal{H}_{A}$ into $\widetilde{\mathcal{H}}$.

We can improve this convergence to $C^{\infty}$ by using the theory of elliptic boundary value problems. The convergence of the $H_{j}$ to $H_{\infty}$ lets us pick a finite number of coordinate charts on $\widetilde{\mathcal{H}}$ so that their pull back to charts on $\mathcal{M}$ by the $\widetilde{F}_{j}$ are such that for any fixed point $X$ in $\mathcal{H}_{A}$ the $F_{j}^{\#}$ take a fixed neighborhood of $X$ for all large $j$ into one of these pull-back charts. In this chart the estimates on lower order derivatives of the $F_{j}^{\#}$ give estimates on the higher order derivatives. On the other hand for large $j$ we can estimate as many derivatives of $\widetilde{F}_{j}$ or $\widetilde{F}_{j}^{-1}$ as we like. Combining these gives uniform 
estimates on all derivatives of $H_{j}=\widetilde{F}_{j}^{-1} \circ F_{j}^{\#}$. It follows that for a better subsequence we can make $H_{j}$ converge to $H_{\infty}$ in $C^{\infty}(\mathcal{H})$.

The limit map will itself be harmonic since the $F_{j}^{\#}$ are harmonic and the $\widetilde{F}_{j}$ are as close as we like to preserving the metric. Moreover we still have

$$
\left\|H_{\infty}^{*} \widetilde{h}-h\right\|_{C^{k}\left(\mathcal{H}_{A}\right)}=\delta
$$

in the limit. Equality here rather than inequality prevents $H_{\infty}$ from being an isometry. The map $H_{\infty}$ will still be a diffeomorphism. It is clear there can be no overlap in the interior, and the boundary cannot have any overlap either because it is strictly concave. Now each $F_{j}^{\#}$ satisfies the constant mean curvature boundary conditions, while $\widetilde{F}_{j}$ is as close to preserving the metric as we like, so the limit $H_{\infty}$ will also satisfy the constant mean curvature boundary conditions. Therefore $H_{\infty}$ takes $\mathcal{H}_{A}$ to $\widetilde{\mathcal{H}}_{A}$.

The rigidity result in Theorem 8.3 guarantees that for $A$ small enough and any $\ell$ and $\varepsilon>0$ we can find $k$ and $\delta>0$ so that the above $H_{\infty}$ guarantees the existence of an isometry $I$ of $\mathcal{H}$ to $\widetilde{\mathcal{H}}$ with

$$
d_{C^{\ell}\left(\mathcal{H}_{A}\right)}\left(H_{\infty}, I\right)<\varepsilon .
$$

In applying rigidity we use the fact that the limit $\mathcal{H}$ has as few cusps as possible, hence no more than $\widetilde{\mathcal{H}}$. We can use $I$ to identify $\widetilde{\mathcal{H}}_{A}$ with $\mathcal{H}_{A}$.

The contradiction comes from observing that we now have two diffeomorphisms, namely $H_{\infty}$ and the identity $I$, of $\mathcal{H}_{A}$ to itself which are close but different, and both are harmonic and satisfy the constant mean curvature boundary conditions. The only way around this contradiction is if one family $F_{j}(t)$ extended to $t \rightarrow \infty$ without a stopping time $t_{j}^{\#}$. This shows at least one hyperbolic piece persists.

We can continue to form other persistent hyperbolic pieces in the same way as long as there are any points $P_{j}$ outside of the chosen pieces where the injectivity radius at times $t_{j} \rightarrow \infty$ are all at least some fixed $\rho>0$. The only modification in the proof is to take the new limit $\mathcal{H}$ to have the least possible number of cusps out of all remaining possible limits.

\section{Variation of Area.}

Now we wish to show that the boundary tori of any persistent hyperbolic piece are incompressible, in the sense that the fundamental group of the torus injects into that of the whole manifold. To see this, we shall assume 
that some curve in the torus bounds a disk in the manifold, and obtain a contradiction.

Let $\mathcal{H}_{B}$ be a hyperbolic piece of the manifold $\mathcal{M}$ truncated by boundary tori of area $B$ with constant mean curvature. We denote by $\mathcal{H}_{B}^{c}=\mathcal{M}-\mathcal{H}_{B}^{\circ}$ the part of $\mathcal{M}$ exterior to $\mathcal{H}_{B}$, i.e. the complement of the interior. By Van Kampen's Theorem, if $\pi_{1}\left(\partial \mathcal{H}_{B}\right)$ injects into $\pi_{1}\left(\mathcal{H}_{B}^{c}\right)$ then it injects into $\pi_{1}(\mathcal{M})$ also. Let $\mathcal{T}$ be a torus in $\partial \mathcal{H}_{B}$. If $\pi_{1}(\mathcal{T})$ does not inject into $\pi_{1}\left(\mathcal{H}_{B}^{c}\right)$, then by Dehn's Lemma the kernel is a cyclic subgroup of $\pi_{1}(\mathcal{T})$ generated by a primitive element. We shall show this leads to a contradiction. The work of Meeks and Yau [M-Y] shows that among all disks in $\mathcal{H}_{B}^{c}$ whose boundary curve lies in $\mathcal{T}$ and generates the kernel, there is a smooth embedded disk normal to the boundary which has the least possible area. Let $A=A(t)$ be the area of this disk. This is defined for all $t$ sufficiently large. What we shall show is that $A(t)$ decreases at a rate bounded away from zero.

Theorem 11.1. For every $\delta>0$ there exists a time $T$ such that for $t \geq T$

$$
\frac{d A}{d t} \leq-(2 \pi-\delta)
$$

in the sense of the lim sup of forward difference quotients.

Now it is clearly intolerable that such a situation should go on forever as $t \rightarrow \infty$, since $A \geq 0$. This contradiction will show that $\pi_{1}(\mathcal{T})$ in fact injects in $\pi_{1}\left(\mathcal{H}_{B}^{c}\right)$ as we desire. Now we turn to the proof of Theorem 11.1.

Let us compute the rate at which $A$ changes under the Ricci flow. We only need show $A$ decreases at least at a certain rate, and since $A$ is the minimum area to bound any disk in the given homotopy class, it will suffice to find some such disk whose area decreases at least that fast. We choose this disk as follows. Pick the minimal disk at time $t_{0}$, and extend it smoothly a little past the boundary torus. For times $t$ a little bigger than $t_{0}$, the boundary torus may need to move a little to stay constant mean curvature with area $B$ as the metric changes, but we leave the surface alone and take the bounding disk to be the one cut off from it by the new torus. There will be two contributions to the change in the area $A$, one from the change in the metric and the other from the change in the boundary.

The change in the metric comes from the normalized Ricci flow

$$
\frac{\partial}{\partial t} g(X, Y)=\frac{2}{3} r g(X, Y)-2 R c(X, Y) .
$$


Choose an orthonormal frame $F_{1}, F_{2}, F_{3}$ at a point $P$ on the surface so that $F_{1}$ and $F_{2}$ are tangent to the surface while $F_{3}$ is normal, and write

$$
R c_{i}=R c\left(F_{i}, F_{i}\right)
$$

for the components of the Ricci tensor. Then the rate of change of the area element $d a$ on the surface is

$$
\frac{\partial}{\partial t} d a=\left[\frac{2}{3} r-R c_{1}-R c_{2}\right] d a,
$$

and the total change of the area of the surface due to the change of the metric $g$ comes from integrating this over the surface. Also the change in area at the boundary from the motion of the boundary with a normal velocity $V$ along a piece of length $d s$ is given by $V d s$, and the total change of the area from the motion if the boundary is given by integrating this over the boundary curve. This gives the formula

$$
\frac{d A}{d t}=\iint\left(\frac{2}{3} r-R c_{1}-R c_{2}\right) d a+\int_{\partial} V d s .
$$

Now write the sectional curvatures as

$$
\begin{aligned}
& R m_{1}=R m\left(F_{2}, F_{3}, F_{2}, F_{3}\right) \\
& R m_{2}=R m\left(F_{3}, F_{1}, F_{3}, F_{1}\right) \\
& R m_{3}=R m\left(F_{1}, F_{2}, F_{1}, F_{2}\right) .
\end{aligned}
$$

Then

$$
\begin{aligned}
& R c_{1}=R m_{2}+R m_{3} \\
& R c_{2}=R m_{1}+R m_{3}
\end{aligned}
$$

and

$$
R c_{1}+R c_{2}=R m_{1}+R m_{2}+2 R m_{3} .
$$

Since

$$
R m_{1}+R m_{2}+R m_{3}=\frac{1}{2} R
$$

where $R$ is the scalar curvature

$$
R c_{1}+R c_{2}=\frac{1}{2} R+R m_{3} .
$$


This gives

$$
\frac{d A}{d t}=\iint\left(\frac{2}{3} r-\frac{1}{2} R\right) d a-\iint R m_{3} d a+\int_{\partial} V d s .
$$

The Gauss curvature of the bounding disk is

$$
K=R m_{3}+\operatorname{det} I I
$$

where det II is the determinant of the second fundamental form II. Since the bounding disk is a minimal surface

$$
\operatorname{det} \text { II } \leq 0 \text {. }
$$

The Gauss-Bonnet Theorem tells us that for a disk

$$
\iint K d a+\int_{\partial} k d s=2 \pi
$$

where $k$ is the geodesic curvature of the boundary. This gives

$$
\frac{d A}{d t} \leq \iint\left(\frac{2}{3} r-\frac{1}{2} R\right) d a+\int_{\partial} k d s+\int_{\partial} V d s-2 \pi .
$$

Consider that for a negative curvature limit under the Ricci flow

$$
r \rightarrow-6 \quad \text { and } \quad \check{R} \rightarrow-6
$$

and $R \geq \check{R}$. Thus for every $\varepsilon>0$ we can find a time $T$ such that for $T \geq t$

$$
\frac{2}{3} r-\frac{1}{2} R \leq-(1-\varepsilon)
$$

and

$$
\iint\left(\frac{2}{3} r-\frac{1}{2} R\right) d a \leq-(1-\varepsilon) A .
$$

The geodesic curvature $k$ of the boundary of the minimal disk is the acceleration of a curve moving with unit speed along the intersection of the disk with the torus; since the disk and the torus are normal, this is the same as the second fundamental form of the torus in the direction of the curve of intersection. Now if the metric were actually hyperbolic, the second fundamental form of the torus would be exactly 1 in all directions. Hence we can find a time $T$ again such that $k \leq 1+\varepsilon$ for $T \geq t$. This makes

$$
\int_{\partial} k d s \leq(1+\varepsilon) L
$$


where $L$ is the length of the boundary curve. Also since the metric is as close to hyperbolic as we like, its change under the Ricci flow is as small as we like; so the motion of the constant mean curvature torus of fixed area $B$ will have a normal velocity $V$ as small as we like. Thus again we can find a time $T$ such that for $t \geq T$ we have $|V| \leq \varepsilon$. This makes

$$
\int_{\partial} V d s \leq \varepsilon L
$$

Combining these estimates, we find the following.

Theorem 11.2. For every $\varepsilon>0$ there is a time $T$ so that for $t \geq T$

$$
\frac{d A}{d t} \leq(1+2 \varepsilon) L-(1-\varepsilon) A-2 \pi
$$

for all $t \geq T$.

In the next section we shall find an estimate saying $L$ is not much bigger than $A$, and this will finish the proof of Theorem 11.1.

\section{Bounding Length by Area.}

It remains to bound the length $L$ of the curve of intersection of the minimal disk with the torus in terms of the area $A$ of the disk. Consider the situation. For large $t$ the metric is as close as we like to hyperbolic; not just on $\mathcal{H}_{B}$ but as far beyond as we like. Thus for a long distance into $\mathcal{H}_{B}^{c}$ the metric will look nearly like a hyperbolic cusplike collar.

We construct a special coordinate system on the cusplike tube projecting beyond the torus $\mathcal{T}$ in $\partial \mathcal{H}_{B}$ as follows. The universal cover of $\mathcal{T}$ can be mapped conformally to the $x-y$ plane so that the deck transformations of $\mathcal{T}$ become translations in $x$ and $y$, and so that the Euclidean area of the quotient is 1 ; then these coordinates are unique up to a translation. Then we extend to a third coordinate $z$, starting with $z=\zeta$ on the torus $\mathcal{T}$ where $\zeta$ is chosen so the torus in the hyperbolic cusp with the same $x$ and $y$ translations at height $\zeta$ has the same area $B$, and so that the lines where both $x$ and $y$ are constant are geodesics perpendicular to $\mathcal{T}$ and the distance $d s=d z / z$ along these geodesics, the same as in hyperbolic space $z>0$ with metric

$$
d s^{2}=\frac{d x^{2}+d y^{2}+d z^{2}}{z^{2}} .
$$

By taking $t$ large we can make the metric in as large a neighborhood of $\mathcal{T}$ as close to hyperbolic as we wish, in the sense that the sectional curvatures 
are as close to -1 and as many covariant derivatives of the curvature as close to zero as we wish; this allows us to make these good coordinates for $\zeta \leq z \leq \zeta^{*}$ for as large a $\zeta^{*}$ as we wish. The translations from $\mathcal{T}$ act as isometries on the set

$$
\left\{\begin{array}{c}
\text { all } x \\
\text { all } y \\
\zeta \leq z \leq \zeta^{*}
\end{array}\right\}
$$

and the resulting quotient maps into $\mathcal{M}$ as above. The metric has the special form

$$
d s^{2}=\frac{g_{x x} d x^{2}+2 g_{x y} d x d y+g_{y y} d y^{2}}{z^{2}}
$$

where $g_{x x}$ and $g_{y y}$ are close to 1 and $g_{x y}$ is close to zero. Moreover since we have bounds on the curvatures and their derivatives, we can make any derivatives of $g_{x x}, g_{x y}$, or $g_{y y}$ as close to zero as we like in these coordinates by taking $t$ large.

Now consider our minimal disk, and let $L(z)$ be the length of the curve of intersection of the disk with the torus at height $z$ in our special coordinates. We prove a monotonicity formula.

12.1 Monotonicity Theorem. For every $\varepsilon>0$ and every $\zeta^{*}$ we can find a time $T$ so that for all $z$ in $\zeta \leq z \leq \zeta^{*}$ and all $t \geq T$ the function

$$
\frac{z^{1+\varepsilon}}{z-\zeta} \int_{\zeta}^{z} \frac{L(w) d w}{w}
$$

is monotone increasing in $z$.

Proof. We construct a comparison disk as follows. For almost every $z$ the intersection of the disk with the torus at height $z$ is a smooth embedded curve or a finite union of them. If there are more curves than one, at least one of them is not homotopic to a point in $\mathcal{T}$, and then it must represent the primitive generator in the kernel of $\pi_{1}(\mathcal{T})$ that dies in $\pi_{1}\left(\mathcal{H}_{B}^{c}\right)$, and in addition part of the original disk beyond height $z$ continues to be a disk that bounds it. We extend this disk back to the initial height $\zeta$ by dropping the curve straight down. Let $\widetilde{L}(z)$ be the length of the curve we picked at height $z$; of course $\widetilde{L}(z) \leq L(z)$ with equality if it is the only piece. Let $\widetilde{L}(w)$ denote the length of the same curve in the $x-y$ plane dropped down 
to height $w$ for $\zeta \leq w \leq z$. We need to be fairly delicate here in estimating the length $\widetilde{L}(w)$. In the hyperbolic space we would have

$$
\widetilde{L}(w)=\frac{z}{w} \widetilde{L}(z)
$$

exactly. In our case there is a small error proportional to $\widetilde{L}(z)$, and we must also take it proportional to the distance $z-w$ by which it drops. This comes from estimating the $z$-derivatives of $g_{x x}, g_{x y}, g_{y y}$ in our special coordinates. Then for every $\delta>0$ and every $\zeta^{*}$ we can pick $T$ so that for $t \geq T$ we have

$$
\left|\widetilde{L}(w)-\frac{z}{w} \widetilde{L}(z)\right| \leq \delta(z-w) \widetilde{L}(z)
$$

for all $z$ and $w$ in $\zeta \leq w \leq z \leq \zeta^{*}$. Now given $\varepsilon$ and $\zeta^{*}$ pick $\delta=2 \varepsilon / \zeta^{*}$. Then

$$
\left|\widetilde{L}(w)-\frac{z}{w} \widetilde{L}(z)\right| \leq \frac{2 \varepsilon z(z-w) \widetilde{L}(z)}{w^{2}}
$$

This makes

$$
\widetilde{L}(w) \leq \frac{z}{w} \widetilde{L}(z)\left[1+\frac{2 \varepsilon(z-w)}{w}\right] .
$$

Now we are ready to do the comparison.

When we drop the curve vertically for our comparison surface we get an area $\widetilde{A}(z)$ between $\zeta$ and $z$ given by the integral of the length $\widetilde{L}(w)$ times the vertical distance $d s=d w / w$, so

$$
\widetilde{A}(z)=\int_{\zeta}^{z} \frac{\widetilde{L}(w) d w}{w} .
$$

On the other hand if we do not drop vertically we pick up even more area, so the area $A(z)$ of the original disk between $\zeta$ and $z$ has

$$
A(z) \geq \int_{\zeta}^{z} \frac{L(w) d w}{w} .
$$

Now the original disk minimized among all disks bounding a curve in the primitive generator of the kernel, and the comparison disk beyond the height $z$ is part of the original disk, so $A(z) \leq \widetilde{A}(z)$. This gives

$$
\int_{\zeta}^{z} \frac{L(w) d w}{w} \leq \int_{\zeta}^{z} \frac{\widetilde{L}(w) d w}{w} .
$$


Now from our comparison of $\widetilde{L}(w)$ to $\widetilde{L}(z) \leq L(z)$ we have

$$
\int_{\zeta}^{z} \frac{\widetilde{L}(w) d w}{w} \leq z L(z) \int_{\zeta}^{z}\left[\frac{1-2 \varepsilon}{w^{2}}+\frac{2 \varepsilon z}{w^{3}}\right] d w
$$

and we evaluate the latter integral as

$$
(1-2 \varepsilon)\left(\frac{1}{\zeta}-\frac{1}{z}\right)+\varepsilon z\left(\frac{1}{\zeta^{2}}-\frac{1}{z^{2}}\right)
$$

which simplifies to

$$
\frac{z-\zeta}{\zeta z}\left[1+\varepsilon\left(\frac{z-\zeta}{\zeta}\right)\right]
$$

This gives the estimate

$$
\int_{\zeta}^{z} \frac{L(w) d w}{w} \leq \frac{z-\zeta}{\zeta}\left[1+\varepsilon\left(\frac{z-\zeta}{\zeta}\right)\right] L(z)
$$

To finish the proof, let

$$
I(z)=\int_{\zeta}^{z} \frac{L(w) d w}{w}
$$

denote the integral. Then

$$
I(z) \leq \frac{z-\zeta}{\zeta}\left[1+\varepsilon\left(\frac{z-\zeta}{\zeta}\right)\right] L(z) .
$$

Using the inequality

$$
\frac{1}{1+x} \geq 1-x
$$

we find that

$$
\frac{\zeta}{z-\zeta}\left[1-\varepsilon\left(\frac{z-\zeta}{\zeta}\right)\right] I(z) \leq L(z)
$$

or equivalently

$$
\left[\frac{\zeta}{z-\zeta}-\varepsilon\right] I(z) \leq L(z)
$$


Thus

$$
\frac{d}{d z} I(z)=\frac{L(z)}{z} \geq\left[\frac{1}{z-\zeta}-\frac{1+\varepsilon}{z}\right] I(z)
$$

and hence

$$
\frac{d}{d z} \log \left\{\frac{z^{1+\varepsilon} I(z)}{z-\zeta}\right\} \geq 0
$$

which shows that

$$
\frac{z^{1+\varepsilon} I(z)}{z-\zeta}
$$

is monotone increasing for $\zeta \leq z \leq \zeta^{*}$ as desired.

Corollary 12.2. We also have

$$
\frac{z^{1+\varepsilon}}{z-\zeta} \int_{\zeta}^{z} \frac{L(w) d w}{w} \geq \zeta^{\varepsilon} L(\zeta)
$$

for $\zeta \leq z \leq \zeta^{*}$

Proof. The quantity on the left is monotone and approaches that on the right as $z \rightarrow \zeta$.

Corollary 12.3. If $A(z)$ is the area of the part of the disk between $\zeta$ and $z$ then

$$
\frac{z^{1+\varepsilon}}{z-\zeta} A(z) \geq \zeta^{\varepsilon} L(\zeta)
$$

Proof. Recall

$$
A(z) \geq \int_{\zeta}^{z} \frac{L(w) d w}{w}
$$

Corollary 12.4. For every $\delta>0$ we can find $\zeta_{\#}$ and $T$ so that for $t \geq T$

$$
L(\zeta) \leq(1+\delta) A\left(\zeta_{\#}\right)
$$


Proof. The previous result gives

$$
L(\zeta) \leq\left(\frac{z}{\zeta}\right)^{\varepsilon} \frac{z}{z-\zeta} A(z)
$$

for all $z \geq \zeta$. Take $z=\zeta_{\#}$ with $\zeta_{\#}$ large so that $z /(z-\zeta)$ is close to 1 , then take $\varepsilon>0$ so small that $(z / \zeta)^{\varepsilon}$ is close to 1 .

Now recall that we had

$$
\frac{d A}{d t} \leq(1+2 \delta) L-(1-\delta) A-2 \pi
$$

where $\delta>0$ is as small as we like, and we only need to show this is negative. Thus for any $\varepsilon>0$ we are in good shape unless

$$
A \leq(1+\varepsilon) L
$$

and hence we can assume

$$
A\left(\zeta^{*}\right) \leq(1+\varepsilon) L(\zeta)
$$

since $A\left(\zeta^{*}\right) \leq A$ and $L(\zeta)=L$. Assuming this now, we proceed. First we show that for some large $z$ the length of $L(z)$ is not too great.

Lemma 12.5. For every $\delta>0$ we can find $T$ and a ratio $r$ so that if $\zeta / 1 \geq r$ and $\zeta_{\#} / \zeta \geq r$ and $\zeta^{*} / \zeta_{\#} \geq r$ then for some $z$ between $\zeta_{\#}$ and $\zeta^{*}$

$$
L(z) \leq(1+\delta) \frac{z}{\zeta^{*}} L(\zeta) .
$$

Proof. Let

$$
X=\inf \left\{\frac{L(z)}{z}: \zeta_{\#} \leq z \leq \zeta^{*}\right\}
$$

Then

$$
\int_{\zeta_{\#}}^{\zeta^{*}} \frac{L(w) d w}{w} \geq\left(\zeta^{*}-\zeta_{\#}\right) X
$$

On the other hand

$$
A\left(\zeta^{*}\right) \geq \int_{\zeta_{\#}}^{\zeta^{*}} \frac{L(w) d w}{w}
$$


and we can assume

$$
A\left(\zeta^{*}\right) \leq(1+\varepsilon) L(\zeta) .
$$

Combining these estimates gives that at the infimum $z$

$$
L(z) \leq \frac{1+\varepsilon}{1-(1 / r)} \frac{z}{\zeta^{*}} L(\zeta)
$$

and we can take $\varepsilon$ and $r$ to depend on $\delta$.

Now we pick another disk for a final comparison. We choose the height $z$ above, where $L(z)$ is not too large, and move the curve there through a small area to agree with the curve in the same homotopy class which is a geodesic circle in the flat metric coming from our special $x-y-z$ coordinates, and then drop this geodesic circle vertically in the special coordinates to complete the disk. Note two technical points. First the curve at the height $z$ where $L(z) / z$ is minimal may not be smooth; this does not matter because the estimate in Lemma 12.5 on $L(z)$ will hold at least on a set of positive measure. Second, if the curve at height $z$ has several pieces, as before one of them will lie in our homotopy class, and we can ignore the others.

Now we given an estimate on the area necessary to deform the curve in the torus.

Theorem 12.6. Given an embedded curve of length $L$ circling the cylinder $S^{1} \times \mathbb{R}$ of circumference $W$ once, it is possible to deform the curve through an area $A \leq L W$ into a meridian circle.

Proof. Since the curve has length $L$, it is contained in a finite cylinder of height $L$. The region between the curve and either end of this finite cylinder is topologically another finite cylinder, and this gives the desired deformation.

Corollary 12.7. Given an embedded curve of length $L$ in a flat torus which generates a non-trivial homotopy class whose geodesic circle generators have length $W$, it is possible to deform the curve through an area $A \leq L W$ into one of these geodesic circles.

Proof. The torus is covered by a cylinder of circumference $W$ where the image of the fundamental group of the cylinder in the fundamental group of the torus is the class of the curve. The embedded curve in the torus lifts to 
an embedded curve in the cylinder. By the previous result we can deform the curve in the cylinder through an area $A \leq L W$ into a meridian circle, and this descends to a deformation of the curve in the torus through no more area into a geodesic circle.

With this estimate on the flat torus we return to the argument. Let $G$ be the length of the geodesic circle in the cusp at height 1 for normalization. Then its length is the hyperbolic metric in the torus at height $z$ will be $G / z$. Our metric and the hyperbolic metric will differ from each other by a factor at most $1+\delta$ where $\delta$ is small for $t$ large. The area in the torus in the flat metric to deform the curve of length $L(z)$ in our metric, which is at most $(1+\delta) L(z)$ in the flat metric, to the geodesic circle of length $G / z$ in the flat metric is bounded by

$$
(1+\delta) G L(z) / z
$$

and the area in our metric will not be greater by more than a factor $(1+\delta)^{2}$. This gives a bound on the area of deformation which is

$$
(1+\delta)^{3} G L(z) / z
$$

The area to drop the geodesic circle from height $z$ to height $\zeta$ in the hyperbolic metric is exactly

$$
G\left(\frac{1}{\zeta}-\frac{1}{z}\right)
$$

and the area in our metric is not greater by more than a factor $(1+\delta)^{2}$. Moreover we can drop $1 / z$ to estimate the area of the vertical column in our metric by

$$
(1+\delta)^{2} G / \zeta
$$

Hence comparing the area of the minimal disk to that of the comparison disk just constructed gives

$$
A(z) \leq(1+\delta)^{3} G\left[\frac{L(z)}{z}+\frac{1}{\zeta}\right] .
$$

But from Lemma 12.5 we have

$$
\frac{L(z)}{z} \leq(1+\delta) \frac{L(\zeta)}{\zeta^{*}}
$$


and from Corollary 12.4 we have

$$
L(\zeta) \leq(1+\delta) A\left(\zeta_{\#}\right)
$$

and $A\left(\zeta_{\#}\right) \leq A(z)$ since $\zeta_{\#} \leq z$. Thus

$$
L(\zeta) \leq(1+\delta)^{5} G\left[\frac{L(\zeta)}{\zeta^{*}}+\frac{1}{\zeta}\right]
$$

Now since $G$ is fixed from the geometry of the limit hyperbolic manifold $\mathcal{H}$ we can make

$$
(1+\delta)^{5} G / \zeta \leq \varepsilon
$$

for any $\varepsilon>0$ by taking $\zeta$ large. This enables us to take $L(\zeta)$ as small as we like by making $\zeta$ large. Thus for any $\varepsilon>0$ we can choose $\zeta$ and $T$ so that for $t \geq T$ we have $L(\zeta) \leq \varepsilon$. But in this case the formula

$$
\frac{d A}{d t} \leq(1+2 \delta) L-(1-\delta) A-2 \pi
$$

works since $(1+2 \delta) L$ is very small and we still have the good term $-2 \pi$. This finishes the proof.

Acknowledgement. The author would like to thank Huai-Dong Cao for correcting the derivation of the ordinary differential inequality in Theorem 4.1 .

\section{References.}

[C-G] J. Cheeger and M. Gromov, Collapsing Riemannian manifolds while keeping their curvature bounded, I, II, J. Differential Geom. 23 (1986) 309-346, 32 (1990) 269-298.

[G-L] M. Gromov and H. B. Lawson, Spin and scalar curvature in the presence of a fundamental group. I, Ann. of Math. 111 (1980) 209-230.

[H1] R. S. Hamilton, Three-manifolds with positive Ricci curvature, J. Differential Geom. 17 (1982) 255-306.

[H2] R. S. Hamilton, Four-manifolds with positive curvature operator, J. Differential Geom. 24 (1986) 153-179.

[H3] R. S. Hamilton, The Ricci flow on surfaces, Contemporary Mathematics 71 (1988) 237-261. 
[H4] R. S. Hamilton, The formation of singularities in the Ricci flow, Surveys in Differential Geometry 2 (1995) 7-136, International Press.

[H5] R. S. Hamilton, Four-manifolds with positive isotropic curvature, to appear in Comm. Anal. Geom.

[H6] R. S. Hamilton, A compactness property for solutions of the Ricci flow, Amer. J. Math. 117 (1995) 545-572.

[H-I] R.S. Hamilton and J. Isenberg, Quasi-convergence of Ricci flow for a class of metrics, Comm. Anal. Geom. 1 (1993) 543-559.

[I] T. Ivey, Ricci solitons on compact three-manifolds, Diff. Geom. Appl. 3 (1993) 301-307.

[K] N. Koiso, On rotationally symmetric Hamilton's equation for KählerEinstein metrics, Recent Topics in Diff. Anal. Geom., Adv. Studies Pure Math., 18-I (1990) 327-337.

[M-Y] W. W. Meeks, III and S.-T. Yau, The existence of embedded minimal surfaces and the problem of uniqueness, Math. Zeit. 179 (1982) 151-168.

[S-Y] R. Schoen and S.-T. Yau, Existence of incompressible minimal surfaces and the topology of three dimensional manifolds with non-negative scalar curvature, Ann. of Math. 110 (1979) 127-142.

RECEIVED MARCH 21, 1996.

Department of MATHEMATics

University of CALIFornia, SAN Diego

LA Jolla, CA 92093-0112 